\title{
Analysis of Neuropathic Pain Using Magnetic Resonance Imaging T2 Mapping of Intervertebral Disc in Chronic Low Back Pain
}

\author{
Izaya Ogon ${ }^{1}$, Tsuneo Takebayashi ${ }^{2}$, Hiroyuki Takashima ${ }^{1}$, Tomonori Morita ${ }^{1}$, Noriyuki Iesato ${ }^{1}$, \\ Katsumasa Tanimoto ${ }^{1}$, Yoshinori Terashima ${ }^{1}$, Mitsunori Yoshimoto ${ }^{1}$, Toshihiko Yamashita ${ }^{1}$ \\ ${ }^{1}$ Department of Orthopaedic Surgery, Sapporo Medical University School of Medicine, Sapporo, Japan \\ ${ }^{2}$ Department of Orthopaedic Surgery, Sapporo Maruyama Orthopaedic Hospital, Sapporo, Japan
}

\begin{abstract}
Study Design: Cross sectional study.
Purpose: The study aimed to analyze mechanisms underlying chronic low back pain (CLBP) using magnetic resonance imaging (MRI) T2 mapping of the intervertebral disc (IVD).

Overview of Literature: MRI T2 mapping utilizes the T2 values for quantifying moisture content and collagen sequence breakdown. We previously used MRI T2 mapping for quantifying the extent of IVD degeneration (IVDD) and showed a correlation between the degeneration of the posterior annulus fibrosus (AF) and CLBP.

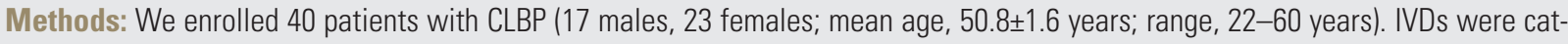
egorized as the anterior AF, nucleus pulposus (NP), and posterior AF, and T2 value for each disc was measured. T2 values, assessed using the Japanese neuropathic pain (NeP) screening questionnaire, of the NeP and nociceptive pain (NocP) groups were compared. Results: T2 values of the NocP and NeP groups were $64.7 \pm 5.6 \mathrm{~ms}$ and $58.1 \pm 2.3 \mathrm{~ms}$ for the anterior AF; $67.0 \pm 4.6 \mathrm{~ms}$ and $59.6 \pm 2.1 \mathrm{~ms}$ for NP; and $70.7 \pm 4.6 \mathrm{~ms}$ and $51.0 \pm 1.2 \mathrm{~ms}$ for the posterior $\mathrm{AF}$, respectively. T2 values for IVDD were significantly lower in the NeP group than those in the NocP group $(p<0.01)$.

Conclusions: The results indicate a correlation between the degeneration of posterior AF and NeP. MRI T2 mapping may be beneficial for detecting NeP caused by IVDD and can help formulate targeted analgesic therapies.
\end{abstract}

Keywords: Intervertebral disc; Low back pains; Neuropathic pain; Magnetic resonance imaging

\section{Introduction}

Low back pain (LBP), a frequently observed condition, is one of the most serious physiological issues worldwide [1]. Various problems in any part of the complex such as the interconnected network of spinal muscles, bones, discs, nerves, or tendons in the lumbar spine can cause
LBP. Intervertebral disc (IVD) degeneration (IVDD) is considered to be the primary condition causing LBP [2]. Many studies have reported on discogenic LBP considering its pathology, diagnosis, and treatment; however, the underlying mechanism and treatments are still to be elucidated. Reportedly, discography is used for supporting the diagnosis of discogenic pain; however, it has a high false-

Received Jun 23, 2018; Revised Aug 23, 2018; Accepted Sep 18, 2018

Corresponding author: Izaya Ogon

Department of Orthopaedic Surgery, Sapporo Medical University School of Medicine, 291, Soutn-1, West-16, Chuo-ku, Sapporo, 0608543, Japan

Tel: +81-11-611-2111, Fax: +81-11-641-6026, E-mail: ogon.izaya@sapmed.ac.jp 
positive rate [3] and is associated with risks that accelerate IVDD [4].

Pain symptoms including LBP are classified into two broad categories based on the mechanism of pain: neuropathic pain (NeP) caused by nervous system injury and nociceptive pain (NocP) caused by tissue-injury and/or inflammation $[5,6]$. Since different type of pains require different treatment approaches [7], early identification of $\mathrm{NeP}$ involvement in patients with chronic pain is crucial for appropriate management [8].

Magnetic resonance imaging (MRI) is an important modality for diagnosing degenerative IVD. Signal variation of the discs observed on T2-weighted images corresponds to the disc age and degeneration and allows for the determination the extent of disc degeneration. Specifically, since MRI signal strength is associated with moisture and proteoglycan content, changes in the MRI signal strength of the nucleus pulposus (NP) can indicate IVDD $[9,10]$. IVDD has been classified using T2-weighted images using the classification system described by Pfirrmann et al. [11], but since this classification is based on visual evaluation, quantifying disc degeneration using this strategy is unclear. Recently, several attempts have been made to utilize MRI T2 and MRI T1p mappings for quantifying lumbar disc degeneration [12-15]. MRI T2 mapping utilizes T2 relaxation time for quantifying moisture content and collagen sequence breakdown. Previously, we used MRI T2 mapping for quantifying the extent of IVDD and demonstrated a significant correlation using the Pfirrmann classification [14]. Furthermore, we quantitatively evaluated IVDD with MRI T2 mapping and demonstrated a correlation between the degeneration of the posterior annulus fibrosus (AF) and chronic LBP (CLBP) [15].

Thus, this study aimed to analyze pain mechanisms underlying discogenic LBP using MRI T2 mapping.

\section{Materials and Methods}

The institutional review board of the Sapporo Medical University approved this study (IRB approval no., 2621074). All subjects were provided with written and verbal explanations of the study prior to obtaining their consent before participating in the study.

\section{Participants}

This study enrolled patients with non-specific CLBP, which was defined as pain, stiffness, and discomfort of the lower back from the 12th rib to the lumbar or lumbosacral area, whose source was difficult to identify, and whose symptoms persisted even following conservative treatment including medication or therapeutic exercise for $>3$ months. Patients with neoplasm, infection, fracture, or a history of lumbar vertebral surgery were excluded. Additionally, patients with neurologic symptoms of the lower leg or obvious instability, which could be identified as a source of LBP and may improve with surgical treatment, were excluded. Intervertebral instability was defined as a sagittal posterior opening of $>5^{\circ}$ on flexion/extension radiographs or a translation of $>3 \mathrm{~mm}$ or segmental motion of $>20^{\circ}$. The severity of LBP was assessed using the Visual Analog Scale (VAS, 0-100) and Japanese NeP Screening Questionnaire after 4 weeks of a washout period of at least 4 weeks [16] (Table 1). Patients with a Japanese NeP Screening Questionnaire score of $\geq 4$ and $<4$ points were categorized in the $\mathrm{NeP}$ and NocP groups, respectively.

Standard VAS scores for diagnosing patients with LBP are not clearly defined. A previous study diagnosed LBP when the VAS score of patients was $>30 \mathrm{~mm}$; therefore, in this study, patients with VAS scores were $>30 \mathrm{~mm}$ were diagnosed with LBP. Patients underwent MRI to detect

Table 1. Questions on the nature of pain

\begin{tabular}{lcccccc|}
\hline Question & None & Slight & Moderate & Severe & Very severe \\
\hline Q1. Pinprick-like pain & 1 & 1 & 1 & 1 & 0 \\
\hline Q2. Electric shock-like pain & 0 & 0 & 0 & 0 & 1 \\
\hline Q3. Tingling burning pain & 0 & 1 & 1 & 1 & 1 \\
\hline Q4. Pain with strong numbness & 0 & 1 & 1 & 1 & 1 \\
\hline Q5. Only a light touch with clothing or cold wind causes a pain & 0 & 1 & 3 & 3 & 3 \\
\hline Q6. Site of pain has decreased or increased sensation & 0 & 1 & 1 & 1 & 1 \\
\hline 07. Site of pain shows skin swelling and/or discoloration to red or purple & 0 & 0 & 0 & 1 & 1 \\
\hline
\end{tabular}


disc degeneration. The clinical radiologist examined and graded MRI T2-weighted images of the five lumbar discs in the midsagittal section as I to $\mathrm{V}$ using the Pfirrmann classification [11]. Patients who had disc degeneration at L4-5 level of grade III to V were screened. Other disc degenerations were graded I to II, as it is likely that L4-5 disc degenerations are the source of discogenic LBP. Finally, 40 patients ( 17 males, 23 females; mean age, $50.8 \pm 1.6$ years; range, $22-60$ years) were enrolled. The distribution of T2-weighted image-based Pfirrmann grade classifications was as follows: grade III, 16 discs; grade IV, 21 discs; and grade $\mathrm{V}, 3$ discs.

\section{Magnetic resonance imaging T2 mapping}

In this study, previously described MRI protocol and analysis methods were used for MRI T2 mapping [14,15]. MRI examinations were performed using a 1.5 Tesla wholebody scanner (Signa HDxt; GE Healthcare, Milwaukee, WI, USA) using a spine coil. Briefly, a T2 map was created using T2 values in the midsagittal section from sagittal sections centered on the lumbar midline region with an
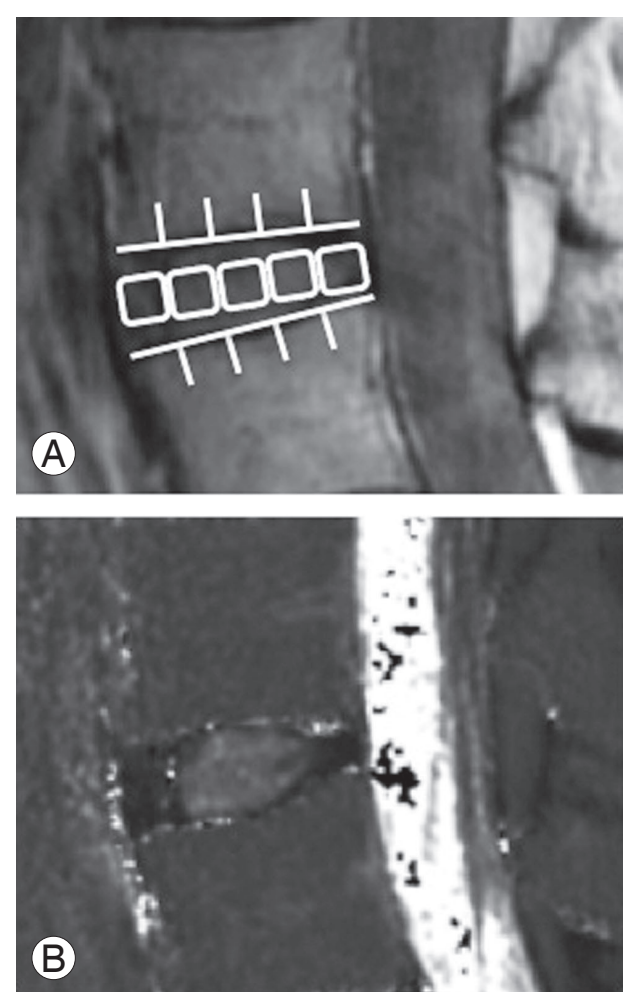

Fig. 1. In the second-echo image, the disc was divided into five areas, designating the front as the anterior $\mathrm{AF}$, the middle as the nucleus pulposus, and the last as the posterior AF (A). Mean T2 values in this region were measured (B). AF, annulus fibrosus. optimized 8 echo multi-spin echo (repetition time/first echo time [TE], 1,000/14.8; last echo TE, 118.6; receiver bandwidth, $\pm 15.63 \mathrm{kHz}$; field-of-view, $22 \mathrm{~cm}$; matrix, $320 \times 256$; slice thickness/gap, $4 \mathrm{~mm} / 4 \mathrm{~mm}, 5$ slices; number of excitations, 2 ; total scan time, 8 minutes and $34 \mathrm{sec}-$ onds) obtained using the Advantage Workstation (version 4.4, Functool; GE Healthcare). However, first echo from the multi-spin system was excluded so as to minimize the effect of the stimulated echo. The T2 map was determined in each pixel from the signal intensity (SI) in the respective TE using the following formula: SI 1/4 e_TE=T2.

For assessment, the IVD was divided into five equal areas based on previous studies: the front fifth was designated as the anterior AF, the middle fifth as the NP, and the last fifth as the posterior AF $[14,15]$. Mean T2 values in the region of interest (ROI) were measured by a researcher (H.T.) using the MedCalc ver. 10.2.0.0 (MedCalc Software, Mariakerke, Belgium) (Fig. 1). The number of pixels for the selected ROI was a minimum of 152 and a maximum of 416. Second-echo images of MRI T2 mapping of patients with NocP and those with NeP are presented in Fig. 2A and 2B, respectively.

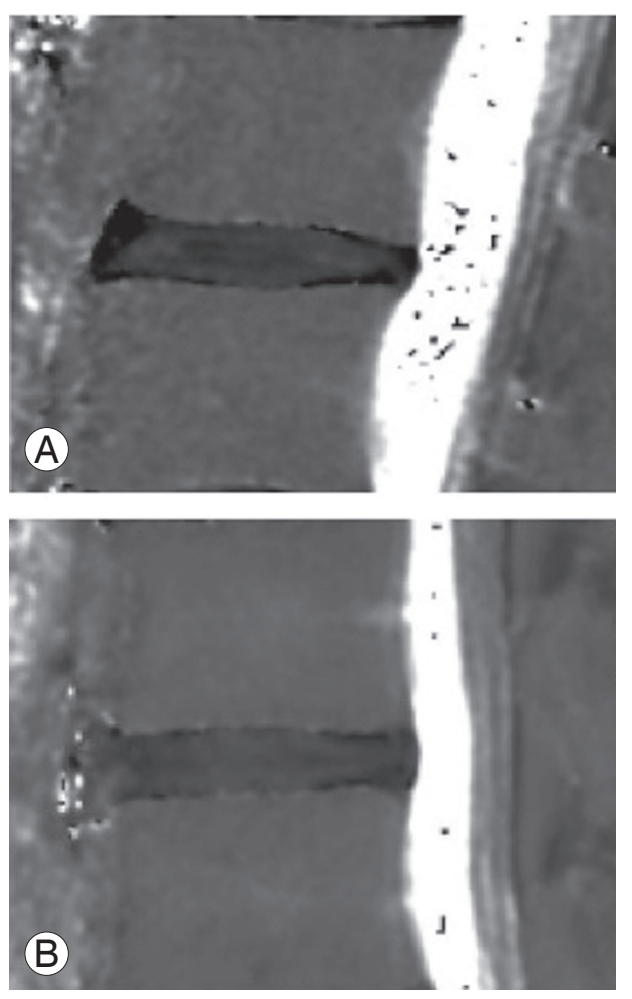

Fig. 2. The second-echo image of magnetic resonance imaging $T 2$ mapping of patients with nociceptive pain (A) and those with neuropathic pain (B). 


\section{Statistical analysis}

T2 values of the $\mathrm{NeP}$ and NocP groups were compared. Demographic data concerning the number of males and females were analyzed using the chi-square test. Differences among groups were compared using the MannWhitney $U$-test. A $p$-value of $<0.05$ indicated statistical significance. All numerical data are expressed as mean \pm standard error of the mean.

\section{Results}

As shown in Table 2 and Fig. 3, the patients were categorized into the NocP $(n=25)$ and $\mathrm{NeP}(\mathrm{n}=15)$ groups. The study cohort comprised an almost equal number of males and females, with no significant difference $(p=0.80)$. Moreover, mean age was not statistically significant between the groups $(p=0.62)$. Average values of $\mathrm{NeP}$ scores were $2.1 \pm 0.2$ and $5.8 \pm 0.3$ in the NocP and NeP groups, respectively.

$\mathrm{T} 2$ values for IVD in the NocP and NeP groups are shown in Fig. 4. T2 values for the NocP and NeP groups were $64.7 \pm 5.6 \mathrm{~ms}$ and $58.1 \pm 2.3 \mathrm{~ms}$ for the anterior AF (Fig.

Table 2. Patient demographic data concerning the sex, age, NeP score

\begin{tabular}{lccc} 
Characteristic & NocP $(n=25)$ & $\operatorname{NeP}(n=15)$ & $p$-value \\
Sex (male:female) & $11: 14$ & $6: 9$ & $0.80^{\text {a) }}$ \\
Age (yr) & $49.3 \pm 2.0$ & $52.4 \pm 1.4$ & $0.62^{\text {b) }}$ \\
NeP score (points) & $2.1 \pm 0.2$ & $5.8 \pm 0.3$ & $<0.01^{\text {b) }}$ \\
\hline
\end{tabular}

Values are presented as mean \pm standard error of the mean.

NeP, neuropathic pain, NocP, nociceptive pain.

alBy chi-square test. ${ }^{b / B y}$ Mann-Whitney U-test.
$4 \mathrm{~A}$ ), $67.0 \pm 4.6 \mathrm{~ms}$ and $59.6 \pm 2.1 \mathrm{~ms}$ for NP (Fig. 4B), and $70.7 \pm 4.6 \mathrm{~ms}$ and $51.0 \pm 1.2 \mathrm{~ms}$ for the posterior $\mathrm{AF}$, respectively (Fig. 4C). T2 values for IVD were significantly lower in the NeP group than those in the NocP group for the posterior $\mathrm{AF}(p<0.01)$

\section{Discussion}

Non-specific LBP is estimated to account for $>85 \%$ of all LBP cases [1]. IVDD was previously considered to be the source of non-specific LBP. Schwarzer et al. [17] suggested that $39 \%$ of CLBP patients had a discogenic origin of their pain. LBP, which was characterized by pain aggravation in a flexure posture, provided an insight to guide diagnosis, but such findings are less objective and specific. Furthermore, the diagnosis of neuropathic components in LBP is more difficult considering that there exists no gold

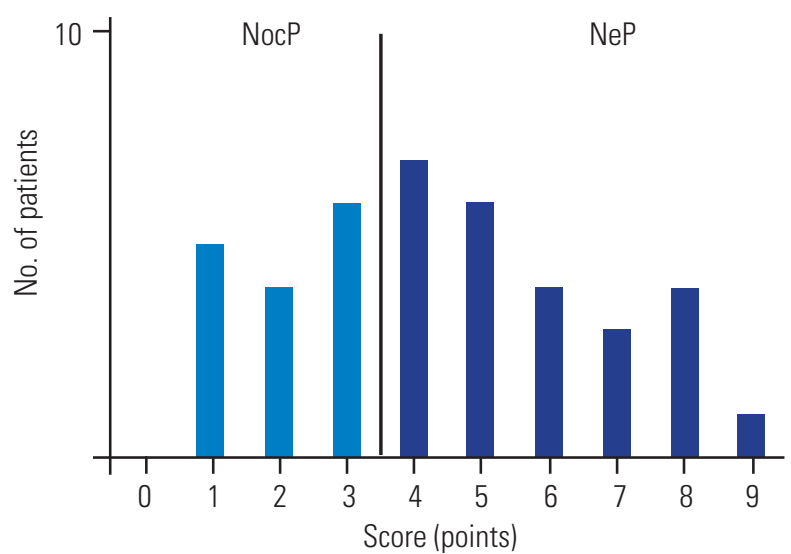

Fig. 3. Distribution of NeP score. Patients were categorized into NocP $(n=25)$ and $\mathrm{NeP}(n=15)$ groups. NeP, neuropathic pain, NocP, nociceptive pain.
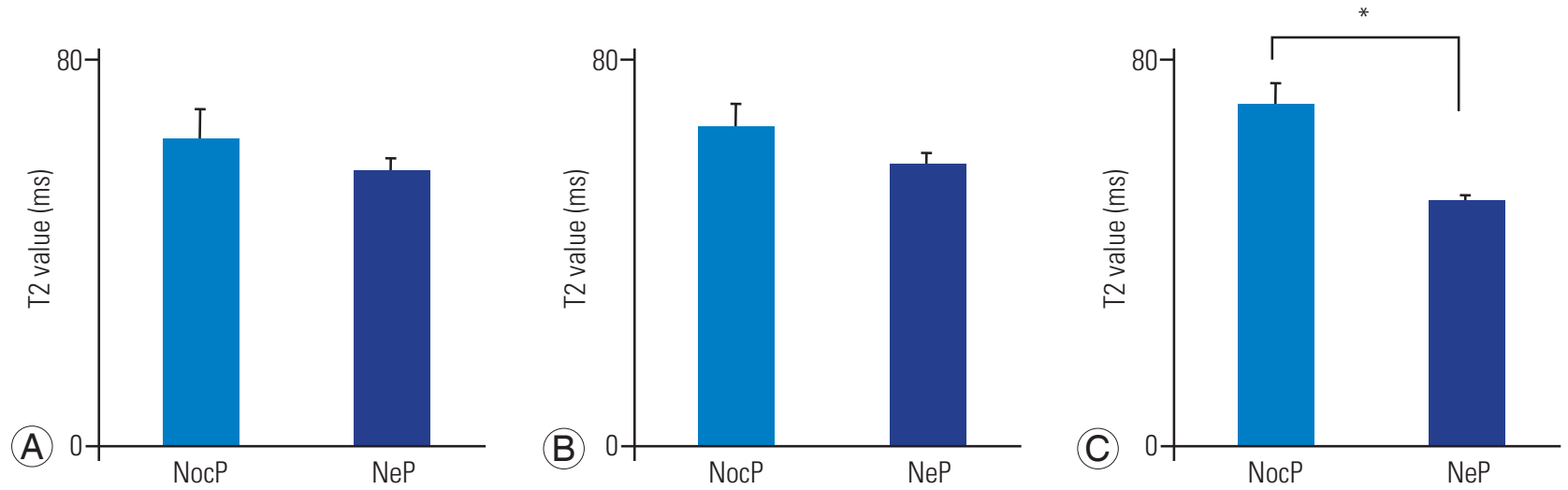

Fig. 4. Comparisons of $T 2$ values for the NocP and NeP groups. T2 values for the anterior $A F(A), N P(B)$, and posterior $A F(C)$ in the NeP group were lower than those in the NocP group; T2 values for the posterior AF significantly differed between the groups. NocP, nociceptive pain; NeP, neuropathic pain; $A F$, annulus fibrosus. ${ }^{*} p<0.01$. 
standard examination for such diagnoses. The diagnostic value of clinical interview, physical examination, and imaging analysis has not been well documented. Previous studies show that $\mathrm{NeP}$ is often underdiagnosed and undertreated [18]. Therefore, no specific diagnostic method has been established; as a result, diagnosis and subsequent treatment of this condition is difficult.

MRI is an important imaging technique for diagnosing structural changes in the spinal column. Recent studies have attempted to quantitatively assess and associate IVDD with LBP using MRI techniques such as MRI T2 mapping and MRI T1p mapping [12-14]. In studies using MR relaxometry, increased disc moisture or glycosaminoglycan content, the primary component of proteoglycan, is associated with increased $\mathrm{T} 2$ values whereas increased collagen content is associated with decreased $\mathrm{T} 2$ values [19]. Recently, some studies have reported that $\mathrm{T} 2$ values for the AF decrease with disc degeneration. Welsch et al. [20] demonstrated that the spatial variation (defined by an increase in $\mathrm{T} 2$ values from the AF to the NP) was the highest for Pfirrmann grade I IVDD and reduced with increasing Pfirrmann grade (II to IV) IVDD. They explained that the AF is composed of fibro-cartilage and functions as a rigid containment for the NP owing to its fibrous structure comprising collagen lamellae and low moisture content. With IVDD, the collagen lamellae of the AF increases in thickness and becomes fibrillated. Therefore, the fibrillated collagen of the AF is associated with a decrease in T2 values. In the present study, we used MRI T2 mapping of IVD in patients with CLBP to quantitatively investigate a difference between the NocP and NeP groups. The results revealed that $\mathrm{T} 2$ values for posterior $\mathrm{AF}$ were significantly lower in the $\mathrm{NeP}$ group than those in the NocP group. Sinuvertebral nerves originate from spinal nerve roots and are distributed around the posterior $\mathrm{AF}$ region. Thus, this area is richly innervated. Freemont et al. [21] reported that the presence of sensory nerve fibers in the deeper layer of the AF may result in discogenic LBP in patients with IVDD. In addition, Kojima et al. [22,23] reported that sinuvertebral nerves innervate both the shallow and deep layers around the posterior longitudinal ligament area. As deep-layer fibers are segmentally located in intervertebral areas of the posterior longitudinal ligament, these fibers control posterior fiber rings. Nakamura et al. [24] reported that when sympathetic nerve trunks were removed from rats, the intervertebral posterior nerve decreased, suggesting that intervertebral posterior nerve fibers pass through the sympathetic nerve trunk. Ohtori et al. [25,26] anteriorly inserted needles with nerve tracers into rat IVDs and demonstrated that the posterior $\mathrm{AF}$ was controlled by sinus spinal and sympathetic nerves. Using rat models of IVD compression and injury, Miyagi et al. [27] demonstrated that repetitive trauma or mechanical stress resulted in a long-lasting increase in the levels of inflammatory mediators in IVD and this increase may eventually lead to the pathogenesis of inflammatory pain and long-lasting increase in calcitonin gene-related peptide expression in the sensory nervous system. This could contribute to pain state, neuronal damage, and nerve regeneration, ultimately aiding $\mathrm{NeP}$ pathogenesis. Lower $\mathrm{T} 2$ values for the posterior AF implied a more severe degeneration. Therefore, results of this study implied that the degeneration of the posterior AF, which is richly innervated with afferent nerve fibers, is more severe in the $\mathrm{NeP}$ group than that in the NocP group.

The prevalence of $\mathrm{NeP}$ was $37.5 \%$ (NocP: $n=25 ; \mathrm{NeP}$ : $\mathrm{n}=15$ ) in this study. A nationwide study in Japan previously reported that $53.3 \%$ of patients with chronic pain related to spine-related disorders presented with $\mathrm{NeP}$, and $29.4 \%$ of patients with LBP were diagnosed with $\mathrm{NeP}$ [28]. This high ratio highlights the necessity to appropriately identify NeP in such patients to facilitate appropriate and early treatment. In clinical settings, the findings of this study have important implications for formulating targeted analgesic therapies. Accurate understanding of the mechanism underlying CLBP and appropriate prescription may result in better patient-oriented pain control. Invasive discography has been conventionally used to evaluate discogenic LBP and can cause pain due to the injection of contrast fluid between IVDs and has a high false-positive rate. Therefore, invasive discography is not entirely suitable as a modality for identifying the source of LBP $[3,29,30]$. Moreover, discography has been reported to cause the progression of IVDD [4]. To the best of our knowledge, there are no reports regarding the efficacy of discography for the diagnosis of NeP. Meanwhile, MRI T2 mapping is a noninvasive quantitative evaluation modality that offers high reproducibility in contrast to discography. The results of the present study suggest that MRI T2 mapping is a quantitative method for diagnosing discogenic pain considering NeP.

This study has several limitations. The Japanese NeP screening questionnaire used in this study may be an inadequate diagnostic utility for detecting $\mathrm{NeP}$ caused 
by spinal disorders [31]. Further studies utilizing an $\mathrm{NeP}$ screening tool that specializes in detecting $\mathrm{NeP}$ caused by spinal disorders such as the Spine painDETECT questionnaire are warranted [32]. Moreover, there is no evidence justifying that non-specific LBP in the patients enrolled in this study originates from L4-5 disc degeneration.

\section{Conclusions}

The results indicate an association between the degeneration of the posterior AF and NeP. This study suggested that MRI T2 mapping is beneficial for detecting NeP caused by IVDD and formulating targeted analgesic therapies.

\section{Conflict of Interest}

No potential conflict of interest relevant to this article was reported.

\section{Author Contributions}

Conception and design: Ogon I, Takebayashi T, Takashima H; acquisition of data: Ogon I, Takashima H; analysis and interpretation of data: Ogon I, Takashima H; drafting the article: Ogon I, Takebayashi T; critically revising the article: all authors; reviewed submitted version of manuscript: all authors; approved the final version of the manuscript on behalf of all authors: Takebayashi T; statistical analysis: Ogon I; administrative/technical/material support: Morita T, Iesato N, Tanimoto K, Terashima Y, Yoshimoto M, Yamashita T; and study supervision: Yamashita T.

\section{References}

1. Deyo RA, Weinstein JN. Low back pain. N Engl J Med 2001;344:363-70.

2. Hurri H, Karppinen J. Discogenic pain. Pain 2004;112:225-8.

3. Carragee EJ, Tanner CM, Yang B, Brito JL, Truong T. False-positive findings on lumbar discography: reliability of subjective concordance assessment during provocative disc injection. Spine (Phila Pa 1976) 1999;24:2542-7.

4. Carragee EJ, Don AS, Hurwitz EL, Cuellar JM, Carrino JA, Herzog R. 2009 ISSLS Prize winner: does discography cause accelerated progression of degenera- tion changes in the lumbar disc: a ten-year matched cohort study. Spine (Phila Pa 1976) 2009;34:2338-45.

5. Woolf CJ, Bennett GJ, Doherty M, et al. Towards a mechanism-based classification of pain? Pain 1998;77:227-9.

6. Freynhagen R, Baron R, Tolle T, et al. Screening of neuropathic pain components in patients with chronic back pain associated with nerve root compression: a prospective observational pilot study (MIPORT). Curr Med Res Opin 2006;22:529-37.

7. Baron R, Binder A, Wasner G. Neuropathic pain: diagnosis, pathophysiological mechanisms, and treatment. Lancet Neurol 2010;9:807-19.

8. Haanpaa ML, Backonja MM, Bennett MI, et al. Assessment of neuropathic pain in primary care. Am J Med 2009;122:S13-21.

9. Urban JP, McMullin JF. Swelling pressure of the lumbar intervertebral discs: influence of age, spinal level, composition, and degeneration. Spine (Phila Pa 1976) 1988;13:179-87.

10. Zou J, Yang H, Miyazaki M, et al. Dynamic bulging of intervertebral discs in the degenerative lumbar spine. Spine (Phila Pa 1976) 2009;34:2545-50.

11. Pfirrmann CW, Metzdorf A, Zanetti M, Hodler J, Boos N. Magnetic resonance classification of lumbar intervertebral disc degeneration. Spine (Phila $\mathrm{Pa}$ 1976) 2001;26:1873-8.

12. Perry J, Haughton V, Anderson PA, Wu Y, Fine J, Mistretta $C$. The value of $\mathrm{T} 2$ relaxation times to characterize lumbar intervertebral disks: preliminary results. AJNR Am J Neuroradiol 2006;27:337-42.

13. Zuo J, Joseph GB, Li X, et al. In vivo intervertebral disc characterization using magnetic resonance spectroscopy and T1 $\rho$ imaging: association with discography and Oswestry Disability Index and Short Form36 Health Survey. Spine (Phila Pa 1976) 2012;37:21421.

14. Takashima H, Takebayashi T, Yoshimoto M, et al. Correlation between $\mathrm{T} 2$ relaxation time and intervertebral disk degeneration. Skeletal Radiol 2012;41:1637.

15. Ogon I, Takebayashi T, Takashima H, et al. Analysis of chronic low back pain with magnetic resonance imaging T2 mapping of lumbar intervertebral disc. J Orthop Sci 2015;20:295-301.

16. Ogawa S. Development of new screening questionnaire to identify neuropathic components in Japanese 
patients with chronic pain. Pain Clin 2010;31:118794.

17. Schwarzer AC, Aprill CN, Bogduk N. The sacroiliac joint in chronic low back pain. Spine (Phila Pa 1976) 1995;20:31-7.

18. Taylor RS. Epidemiology of refractory neuropathic pain. Pain Pract 2006;6:22-6.

19. Nightingale T, MacKay A, Pearce RH, Whittall KP, Flak B. A model of unloaded human intervertebral disk based on NMR relaxation. Magn Reson Med 2000;43:34-44.

20. Welsch GH, Trattnig S, Paternostro-Sluga T, et al. Parametric T2 and T2* mapping techniques to visualize intervertebral disc degeneration in patients with low back pain: initial results on the clinical use of 3.0 Tesla MRI. Skeletal Radiol 2011;40:543-51.

21. Freemont AJ, Peacock TE, Goupille P, Hoyland JA, O’Brien J, Jayson MI. Nerve ingrowth into diseased intervertebral disc in chronic back pain. Lancet 1997;350:178-81.

22. Kojima Y, Maeda T, Arai R, Shichikawa K. Nerve supply to the posterior longitudinal ligament and the intervertebral disc of the rat vertebral column as studied by acetylcholinesterase histochemistry: I. distribution in the lumbar region. J Anat 1990;169:23746.

23. Kojima Y, Maeda T, Arai R, Shichikawa K. Nerve supply to the posterior longitudinal ligament and the intervertebral disc of the rat vertebral column as studied by acetylcholinesterase histochemistry: II. regional differences in the distribution of the nerve fibres and their origins. J Anat 1990;169:247-55.

24. Nakamura S, Takahashi K, Takahashi Y, Morinaga T, Shimada Y, Moriya H. Origin of nerves supplying the posterior portion of lumbar intervertebral discs in rats. Spine (Phila Pa 1976) 1996;21:917-24.
25. Ohtori S, Takahashi Y, Takahashi K, et al. Sensory innervation of the dorsal portion of the lumbar intervertebral disc in rats. Spine (Phila $\mathrm{Pa} 1976$ ) 1999;24:2295-9.

26. Ohtori S, Takahashi K, Chiba T, Yamagata M, Sameda $\mathrm{H}$, Moriya $\mathrm{H}$. Sensory innervation of the dorsal portion of the lumbar intervertebral discs in rats. Spine (Phila Pa 1976) 2001;26:946-50.

27. Miyagi M, Ishikawa $T$, Kamoda $H$, et al. ISSLS Prize winner: disc dynamic compression in rats produces long-lasting increases in inflammatory mediators in discs and induces long-lasting nerve injury and regeneration of the afferent fibers innervating discs: a pathomechanism for chronic discogenic low back pain. Spine (Phila Pa 1976) 2012;37:1810-8.

28. Yamashita T, Takahashi K, Yonenobu K, Kikuchi S. Prevalence of neuropathic pain in cases with chronic pain related to spinal disorders. J Orthop Sci 2014;19:15-21.

29. Carragee EJ, Paragioudakis SJ, Khurana S. 2000 Volvo Award winner in clinical studies: lumbar high-intensity zone and discography in subjects without low back problems. Spine (Phila Pa 1976) 2000;25:298792.

30. Carragee EJ, Hannibal M. Diagnostic evaluation of low back pain. Orthop Clin North Am 2004;35:7-16.

31. Orita S, Yamashita T, Ohtori S, et al. Prevalence and location of neuropathic pain in lumbar spinal disorders: analysis of 1804 consecutive patients with primary lower back pain. Spine (Phila Pa 1976) 2016;41:1224-31.

32. Nikaido T, Sumitani M, Sekiguchi M, Konno S. The Spine painDETECT questionnaire: development and validation of a screening tool for neuropathic pain caused by spinal disorders. PLoS One 2018;13:e0193987. 